\title{
Real Recursive Functions and Real Extensions of Recursive Functions
}

\author{
Olivier Bournez and Emmanuel Hainry \\ LORIA/INRIA, 615 Rue du Jardin Botanique \\ 54602 Villers-Lès-Nancy, FRANCE \\ \{Olivier.Bournez,Emmanuel.Hainry\}@loria.fr
}

\begin{abstract}
Recently, functions over the reals that extend elementarily computable functions over the integers have been proved to correspond to the smallest class of real functions containing some basic functions and closed by composition and linear integration.

We extend this result to all computable functions: functions over the reals that extend total recursive functions over the integers are proved to correspond to the smallest class of real functions containing some basic functions and closed by composition, linear integration and a very natural unique minimization schema.
\end{abstract}

\section{Introduction}

The power of digital discrete time models of computations is rather well understood: all reasonable and sufficiently powerful digital discrete time models have the same power thanks to Turing's work and so-called Church thesis.

For analog models the situation is far from being so clear. Several models have been defined (e.g. the General Purpose Analog Computer (GPAC) model of Shannon [28], neural network models [29,24], hybrid systems [3,4], or theoretical physic models $[11,15,23], \ldots$ ) but there are only few results concerning relations between their respective computational power: GPAC computable functions have been characterized mathematically as differentially algebraic functions $[12,18,25,28]$ but this does not provide directly a way to understand the relations between the power of such machines compared to classical discrete machines. Several other analog models have been shown to exhibit super-Turing computational power: using the so-called Zeno's paradox, some models make it possible to compute non-Turing computable functions in a constant time: see e.g. [3,5,11,15,19]; the continuity of the space makes it sometimes possible to have models whose power is close to non-uniform complexity classes [29].

Since the progress of electronics and other domains of physics such as mechanics or optics makes the construction of some of the machines realistic, clarifying the situation becomes a crucial matter.

In [19], Moore introduced a class of functions over the reals inspired from the classical characterization of computable functions over integers: observing that 
the continuous analog of a primitive recursion is a differential equation, Moore proposes to consider the class of $\mathbb{R}$-recursive functions, defined as the smallest class of functions containing some basic functions, and closed by composition, differential equation solving (called integration), and minimization. The minimization schema of [19] makes it possible to use a "compression trick" (another incarnation of Zeno's paradox) to simulate in a bounded time an unbounded number of discrete transitions in order to recognize arithmetical (hence nonTuring-computable) reals [19].

Actually, the original definitions of [19] suffer from several technical problems that appear as soon as the minimization schema is used (see e.g. discussions in $[19,9,10,20,21])$, and it has been proposed to replace minimization schema by a limit schema to have well-defined classes of functions as in [20,21], or to restrict to functions defined without minimization schema as in $[10,12]$.

Concerning second approach, in his $\mathrm{PhD}$ dissertation [10], Campagnolo proposes to consider a class $\mathcal{L}$ of real-functions built in analogy with the class of elementarily computable functions in classical discrete computability: class $\mathcal{L}$ is defined as the smallest class of functions containing some well-chosen basic functions and closed by composition and linear integration.

Class $\mathcal{L}$ is proved by Campagnolo et al. to be related to functions elementarily computable over the integers in classical recursion theory: any function over the integers elementary in the sense of classical recursion theory is the restriction to integers of a function that belongs to $\mathcal{L}[10,9]$; any function in $\mathcal{L}$ that preserves integers has its restriction to integers elementarily computable $[10,9]$.

This paper proves that this is indeed possible to define a reasonable minimization schema to get a class, that we call $\mathcal{L}+! \mu$, that corresponds in a similar way to all (i.e. not necessarily elementary) computable functions over the integers: we prove that any total recursive function over the integers is the restriction to integers of a function that belongs to $\mathcal{L}+$ ! $\mu$, and that any function in $\mathcal{L}+$ ! $\mu$ that preserves integers has its restriction to integers total recursive.

Concerning, classical discrete computability, we get a new original characterization of computable functions in terms of restrictions to integers of a natural class of functions over the reals.

Concerning analog models, our results relate the computational power of some algebraically defined classes of functions over the reals to classical discrete models, and hence contribute to understand computations over the reals, or at least to understand the computational power of $\mathbb{R}$-(sub)-recursive functions.

Furthermore the problem we solve is in some sense the definition of a minimization operator, which is strong enough to get at least Turing machine power, but not too strong to get the technical problems of [19], nor non-robust superTuring Zeno phenomena of $[3,5,11,15,19]$. In that sense, we believe that our results may be a step toward understanding criteria that could guarantee "robustness" for continuous models as sought by papers like $[2,14]$.

Moreover, we think that that our results could be a first step toward getting an algebraic characterization of functions over the real numbers computable in the sense of recursive analysis, in the spirit of [6], and alternative to $[7,8]$. 


\section{Preliminaries}

\subsection{Mathematical preliminaries}

Let $\mathbb{N}, \mathbb{Q}, \mathbb{R}$, denote the set of natural integers, the set of rational numbers, and the set of real numbers respectively. Given $x \in \mathbb{R}^{n}$, we write $\vec{x}$ to emphasize that $x$ is a vector.

Lemma 1 (Bounding Lemma for Linear Differential Equations (see e.g. [1])). For linear differential equation $\vec{x}^{\prime}=A(t) \vec{x}$, if $A$ is defined and continuous on interval $I=[a, b]$, where $a \leq 0 \leq b$, then, for all $\vec{x}_{0}$, the solution of $\vec{x}^{\prime}=A(t) \vec{x}$ with initial condition $\vec{x}(0)=\vec{x}_{0}$ is defined and unique on $I$. Furthermore, the solution satisfies $\|\vec{x}(t)\| \leq\left\|\vec{x}_{0}\right\| \exp \left(\sup _{\tau \in[0, t]}\|A(\tau)\| t\right)$.

Lemma 2 (Implicit Functions Theorem (see e.g. [26])). Let $f: \mathbb{R}^{k+1} \rightarrow$ $\mathbb{R}$ be a function of class $\mathcal{C}^{k}$, for $k \geq 1$. Assume that for all $\vec{x}$, the equation $f(\vec{x}, y)=0$ has exactly one solution $y$. Assume for all $\vec{x}$ that $\frac{\partial f}{\partial y}(\vec{x}, y) \neq 0$ in the corresponding root $y$. Then function $g: \mathbb{R}^{k} \rightarrow \mathbb{R}$ that maps $\vec{x}$ to the corresponding root $y$ is also of class $\mathcal{C}^{k}$.

\subsection{Classical Recursion Theory}

Classical recursion theory deals with functions over integers. Most classes of classical recursion theory can be characterized as closures of a set of basic functions by a finite number of basic rules to build new functions [27,22]: given a set $\mathcal{F}$ of functions and a set $\mathcal{O}$ of operators on functions (an operator is an operation that maps one or more functions to a new function), $[\mathcal{F} ; \mathcal{O}]$ will denote the closure of $\mathcal{F}$ by $\mathcal{O}$.

Proposition 1 (Classical settings: see e.g. [27,22]). Let $f$ be a function from $\mathbb{N}^{k}$ to $\mathbb{N}$ for $k \in \mathbb{N}$. Function $f$ is

- elementary iff it belongs to $\mathcal{E}=[0, S, U,+, \ominus$; COMP, BSUM, BPROD $]$;

- primitive recursive iff it belongs to $\mathcal{P} \mathcal{R}=[0, U, S ; \mathrm{COMP}, \mathrm{REC}]$;

- total recursive iff it belongs to $\mathcal{R} e c=[0, U, S ; \mathrm{COMP}, \mathrm{REC}, \mathrm{MU}]$.

A function $f: \mathbb{N}^{k} \rightarrow \mathbb{N}^{l}$ is elementary (resp: primitive recursive, total recursive) iff its projections are elementary (resp: primitive recursive, total recursive).

The basic functions $0,\left(U_{i}^{m}\right)_{i, m \in \mathbb{N}}, S,+, \ominus$ and the operators BSUM, BPROD, COMP, REC, MU are given by

1. $0: \mathbb{N} \rightarrow \mathbb{N}, 0: n \mapsto 0 ; U_{i}^{m}: \mathbb{N}^{m} \rightarrow \mathbb{N}, U_{i}^{m}:\left(n_{1}, \ldots, n_{m}\right) \mapsto n_{i} ; S: \mathbb{N} \rightarrow$ $\mathbb{N}, S: n \mapsto n+1 ;+: \mathbb{N}^{2} \rightarrow \mathbb{N},+:\left(n_{1}, n_{2}\right) \mapsto n_{1}+n_{2} ; \ominus: \mathbb{N}^{2} \rightarrow \mathbb{N}$, $\ominus:\left(n_{1}, n_{2}\right) \mapsto \max \left(0, n_{1}-n_{2}\right) ;$

2. BSUM : bounded sum. Given $f, h=\operatorname{BSUM}(f)$ is defined by $h:(\vec{x}, y) \mapsto$ $\sum_{z<y} f(\vec{x}, z)$; $\mathrm{BPROD}:$ bounded product. Given $f, h=\operatorname{BPROD}(f)$ is defined by $h:(\vec{x}, y) \mapsto \prod_{z<y} f(\vec{x}, z)$; 
3. COMP : composition. Given $f_{1}, \ldots, f_{p}$ and $g, h=\operatorname{COMP}\left(f_{1}, \ldots, f_{p} ; g\right)$ is defined as the function verifying $h(\vec{x})=g\left(f_{1}(\vec{x}), \ldots, f_{p}(\vec{x})\right)$;

4. REC : primitive recursion. Given $f$ and $g, h=\operatorname{REC}(f, g)$ is defined as the function verifying $h(\vec{x}, 0)=f(\vec{x})$ and $h(\vec{x}, n+1)=g(\vec{x}, n, h(\vec{x}, n))$.

5. MU : minimization. Given a function $f$ such that for all $\vec{x}$, there is a $y$ with $f(\vec{x}, y)=0$, the minimization of $f$ is $\mu f: \vec{x} \mapsto \inf \{y ; f(\vec{x}, y)=0\}$.

Observe that we consider here only total functions. Furthermore, observe that minimization operator can actually be reinforced into a unique minimization operator as follows:

Proposition 2. A function $f$ from $\mathbb{N}^{k}$ to $\mathbb{N}^{l}$, for $k, l \in \mathbb{N}$, is total recursive iff its projections belong to $[0, U, S ; \mathrm{COMP}, \mathrm{REC}, \mathrm{UMU}]$ where operator $\mathrm{UMU}$ is defined as follows:

1. UMU: unique minimization. Given $f$ such that for all $\vec{x}$ there is a unique $y$ with $f(\vec{x}, y)=0$, the unique minimization of $f$ is defined as the function, denoted by $! \mu(f)(\vec{x}, y)$, that maps $\vec{x}$ to that unique $y$, for all $\vec{x}$.

Proof. The inclusion $[0, U, S ; \mathrm{COMP}, \mathrm{REC}, \mathrm{UMU}] \subset \mathcal{R} e c$ is immediate. Conversely, let $\phi$ be a function from Rec. It is well known [16,27] that $\phi$ can be written as $\phi=\chi \circ \mu(\psi)$ with $\chi$ and $\psi$ in $\mathcal{E}$ and such that for all $\vec{x}$, there is at least a $y$ with $\psi(\vec{x}, y)=0$ (recall that $\phi$ is total). Let $\sigma$ be the elementary function defined by $\sigma(m, n)=\prod_{z<n} \psi(m, z)$. Given $m$, let us note $n_{0}=\mu(\psi)(m)$. We have $\forall n \leq n_{0}, \sigma(m, n) \neq 0$ and $\forall n>n_{0}, \sigma(m, n)=0$. Let $\kappa(m, n)=1 \ominus(1 \ominus((1 \ominus \sigma(m, n))+\sigma(m, n+1)))$. We have clearly $\forall n<n_{0}$, $\kappa(m, n)=1, \kappa\left(m, n_{0}\right)=0$ and $\forall n>n_{0}, \kappa(m, n)=1$, hence $\mu(\kappa)=! \mu(\kappa)=\mu(\psi)$. $\kappa$ is an elementary function and we have $\phi=\chi 0 ! \mu(\kappa)$, hence $\phi$ belongs to $[0, U, S ; \mathrm{COMP}, \mathrm{REC}, \mathrm{UMU}]$.

We have $\mathcal{E} \subseteq \mathcal{P} \mathcal{R} \subseteq \mathcal{R} e c$, and the inclusions are known to be strict $[27,22]$. If $\operatorname{TIME}(t)$ and $\operatorname{SPACE}(t)$ denote the classes of functions that are computable with time and space $t$, then, $\mathcal{P} \mathcal{R}=\operatorname{TIME}(\mathcal{P} \mathcal{R})=\operatorname{SPACE}(\mathcal{P} \mathcal{R})[27,22]$. Class $\mathcal{P R}$ corresponds to functions computable using For-Next programs. Class $\mathcal{E}$ corresponds to computable functions bounded by some iterate of the exponential function $[27,22]$.

In classical computability, more general objects than functions over the integers can be considered, in particular functionals, i.e. functions $\Phi:\left(\mathbb{N}^{m}\right)^{\mathbb{N}} \times \mathbb{N}^{k} \rightarrow$ $\mathbb{N}^{l}$. A functional will be said to be elementary (or primitive recursive, recursive) when it belongs to the corresponding ${ }^{1}$ class.

\footnotetext{
${ }^{1}$ Formally, a function $f$ over the integers can be considered as functional $\bar{f}:(V, \vec{n}) \mapsto$ $f(\vec{n})$. Similarly, an operator $O p$ on functions $f_{1}, \ldots, f_{m}$ over the integers can be extended to argument $\overline{O p}\left(F_{1}, \ldots, F_{m}\right):(V, \vec{n}) \mapsto O p\left(f_{1}(V,),. \ldots, f_{m}(V,).\right)(\vec{n})$.

In that spirit, given some set $\mathcal{F}$ of basic functions $\mathbb{N}^{k} \rightarrow \mathbb{N}^{l}$ and a set $\mathcal{O}$ of operators on functions over the integers, we will still (abusively) denote by $\left[f_{1}, \ldots, f_{p} ; O_{1}, \ldots, O_{q}\right]$ for the smallest class of functionals that contains basic functions $f_{1}, \ldots, f_{p}$, plus the functional Map $:(V, n) \rightarrow V_{n}$, the nth element of sequence $V$, and which is closed by the operators $\overline{O_{1}}, \ldots, \overline{O_{q}}$. For example, a functional will be said elementary iff it belongs to $\mathcal{E}=[\operatorname{Map}, \overline{0}, \bar{S}, \bar{U}, \mp, \bar{\ominus} ; \overline{\mathrm{COMP}}, \overline{\mathrm{BSUM}}, \overline{\mathrm{BPROD}}]$.
} 


\section{Computable Analysis}

The idea sustaining computable analysis, also called recursive analysis, is to define computable functions over real numbers by considering functionals over fast-converging sequences of rationals [30,17,13,31].

Let $\nu_{\mathbb{Q}}: \mathbb{N} \rightarrow \mathbb{Q}$ be the following representation ${ }^{2}$ of rational numbers by integers: $\nu_{\mathbb{Q}}(\langle p, r, q\rangle) \mapsto \frac{p-r}{q+1}$, where $\langle., .,\rangle:. \mathbb{N}^{3} \rightarrow \mathbb{N}$ is a computable bijection.

A sequence of integers $\left(x_{i}\right) \in \mathbb{N}^{\mathbb{N}}$ represents a real number $x$ if $\left(\nu_{\mathbb{Q}}\left(x_{i}\right)\right)$ converges quickly toward $x$ (denoted by $\left(x_{i}\right) \rightsquigarrow x$ ) in the following sense : $\forall i,\left|\nu_{\mathbb{Q}}\left(x_{i}\right)-x\right|<2^{-i}$. For $\left(x_{i}\right) \in\left(\mathbb{N}^{k}\right)^{\mathbb{N}}$, we write $\left(x_{i}\right) \rightsquigarrow x$ when it holds componentwise.

Definition 1 (Recursive analysis [31]). A function $f: \mathbb{R}^{k} \rightarrow \mathbb{R}$ is said computable (or real-computable) if there exists a recursive functional $\Phi:\left(\mathbb{N}^{k}\right)^{\mathbb{N}} \times$ $\mathbb{N} \rightarrow \mathbb{N}$ such that for all $\vec{x} \in \mathbb{R}^{k}$, for all sequence $X=\left(\vec{x}_{n}\right) \in\left(\mathbb{N}^{k}\right)^{\mathbb{N}}$, we have $(\phi(X, j))_{j} \rightsquigarrow f(\vec{x})$ whenever $X \rightsquigarrow \vec{x}$. A function $f: \mathbb{R}^{k} \rightarrow \mathbb{R}^{l}$, with $l>1$, is said computable if all its projections are.

A function $f$ will be said elementarily computable whenever the corresponding functional $\Phi$ is. The class of computable (respectively elementarily computable) functions over the reals will be denoted by $\operatorname{Rec}(\mathbb{R})(\operatorname{resp} . \mathcal{E}(\mathbb{R}))$.

\section{Real-sub-recursive and sub-recursive functions}

Following the original ideas from [19], but avoiding the minimization schema of [19] source of many problems, Campagnolo proposed in [10] to consider the following class, built in analogy with elementarily computable functions over the integers.

Definition $2([\mathbf{1 0}, \mathbf{9}])$. Let $\mathcal{L}$ be the class of functions $f: \mathbb{R}^{k} \rightarrow \mathbb{R}^{l}$, for some $k, l \in \mathbb{N}$, defined by $\mathcal{L}=\left[0,1,-1, \pi, U, \theta_{3} ; \mathrm{COMP}, \mathrm{LI}\right]$ where the basic functions $0,1,-1, \pi,\left(U_{i}^{m}\right)_{i, m \in \mathbb{N}}, \theta_{3}$ and the schemata COMP and LI are the following:

1. $0,1,-1, \pi$ are the corresponding constant functions; $U_{i}^{m}: \mathbb{R}^{m} \rightarrow \mathbb{R}$ are, as in the classical settings, projections: $U_{i}^{m}:\left(x_{1}, \ldots, x_{m}\right) \mapsto x_{i}$;

2. $\theta_{3}: \mathbb{R} \rightarrow \mathbb{R}$ is defined as $\theta_{3}: x \mapsto x^{3}$ if $x \geq 0$, 0 otherwise;

3. COMP: composition is defined as in the classical settings: Given $f_{1}, \ldots, f_{p}$ and $g, h=\operatorname{COMP}\left(f_{1}, \ldots, f_{p} ; g\right)$ is defined by $h(\vec{x})=g\left(f_{1}(\vec{x}), \ldots, f_{p}(\vec{x})\right)$;

4. LI: linear integration. From $g$ and $h, \mathrm{LI}(g, h)$ is the maximal solution of the linear differential equation $\frac{\partial f}{\partial y}(\vec{x}, y)=h(\vec{x}, y) f(\vec{x}, y)$ with $f(\vec{x}, 0)=$ $g(\vec{x})$.

In this schema, if $g$ goes to $\mathbb{R}^{n}, f=\mathrm{LI}(g, h)$ also goes to $\mathbb{R}^{n}$ and $h(\vec{x}, y)$ is a $n \times n$ matrix with elements in $\mathcal{L}$.

\footnotetext{
${ }^{2}$ Many other natural representations of rational numbers can be chosen and provide the same class of computable functions: see [31].
} 
Class $\mathcal{L}$ includes common functions like $+, \sin , \cos ,-, \times, \exp$, or $x \rightarrow r$ for all $r \in \mathbb{Q}$ (see $[10,9])$, but contains only total functions [9]:

Proposition 3 ([9]). All functions from $\mathcal{L}$ are continuous, defined everywhere, and of class $\mathcal{C}^{2}$.

Actually, observing the proofs from $[10,9]$, schema LI can be strengthened as follows:

Proposition 4. Class $\mathcal{L}$ is also the class of functions $f: \mathbb{R}^{k} \rightarrow \mathbb{R}^{l}$, for some $k, l \in \mathbb{N}$, defined by $\mathcal{L}=\left[0,1,-1, \pi, U, \theta_{3} ; \mathrm{COMP}, \mathrm{CLI}\right]$ where $\mathrm{CLI}$ is the following schema:

1. CLI: controlled linear integration. From $g$ and $h$, and $c$, with $h$ differentiable and entries of $h^{\prime}$ bounded by $c, \operatorname{CLI}(g, h, c)$ is the maximal solution of the linear differential equation $\frac{\partial f}{\partial y}(\vec{x}, y)=h(\vec{x}, y) f(\vec{x}, y)$ with $f(\vec{x}, 0)=g(\vec{x})$.

In this schema, if g goes to $\mathbb{R}^{n}, f=\operatorname{CLI}(g, h, c)$ also goes to $\mathbb{R}^{n}$ and $h(\vec{x}, y)$ is a $n \times n$ matrix with elements in $\mathcal{L}$.

Class $\mathcal{L}$ can be related to the class $\mathcal{E}$ of elementarily computable functions over the integers. A real extension $\tilde{f}$ of a function $f: \mathbb{N}^{k} \rightarrow \mathbb{N}^{l}$ over the integers is a function $\tilde{f}$ from $\mathbb{R}^{k}$ to $\mathbb{R}^{l}$ whose restriction to $\mathbb{N}^{k}$ is $f$. Observe that a function $\tilde{f}: \mathbb{R}^{k} \rightarrow \mathbb{R}^{l}$ over the reals is an extension of a function over the integers iff its preserves integers: $\tilde{f}\left(\mathbb{N}^{k}\right) \subset \mathbb{N}^{l}$.

Definition 3 (Discrete Part). Given a class $\mathcal{C}$ of real functions, we denote by $D P(\mathcal{C})$ the class of functions over the integers that have a real extension in $\mathcal{C}$.

Proposition $5([\mathbf{1 0}, \mathbf{9}]) . \mathcal{E}=D P(\mathcal{L})$. I.e.:

- If a function from $\mathcal{L}$ extends some functions over the integers, this latter function is elementarily computable.

- Any elementarily computable function over the integers, has a real extension that belongs to $\mathcal{L}$.

Actually, class $\mathcal{L}$ can also be partially related to the class $\mathcal{E}(\mathbb{R})$ of functions over the real numbers elementarily computable in the sense of recursive analysis: any function from $\mathcal{L}$ is in $\mathcal{E}(\mathbb{R})[10,9]$. We proved in [6] that the inclusion is actually strict, but that adding a limit schema to class $\mathcal{L}$, allows us to capture whole class $\mathcal{E}(\mathbb{R})$ for functions defined over a compact domain.

\section{Real-recursive and recursive functions}

We are now going to extend the class $\mathcal{L}$ with a minimization schema in order to get a class whose discrete part correspond to total recursive functions over the integers.

To do so, we need to introduce a zero-finding operator that permits to simulate the classical discrete minimization schema over the integers. However, this 
operator needs to be stricter than a simple "return the smallest root" since this idea, investigated in [19], has shown to be the source of numerous problems, including ill-defined problems and super-Turing Zeno phenomena $[10,9,21,20,19]$.

Our idea is to use the alternative UMU schema which is equivalent to schema MU for classical computability, but has real counterparts which turn out to preserve real computability.

Indeed, motivated by Proposition 2, by Lemma 2, and by results from recursive analysis about the computability of zeros (see e.g. [31]), we define our unique-zero-finding operator UMU as follows (observe that we also take schema CLI instead of schema LI, which is equivalent when schema UMU is not present):

Definition 4. Given a differentiable function $f$ from $\mathbb{R}^{k+1}$ to $\mathbb{R}$, if for all $\vec{x}, y \mapsto f(\vec{x}, y)$ is a non-decreasing function with a unique root $y_{0}$, on which $\frac{\partial f}{\partial y}\left(\vec{x}, y_{0}\right)>0$, then $\operatorname{UMU}(f)$ is defined as follows:

$$
\mathrm{UMU}(f):\left\{\begin{array}{l}
\mathbb{R}^{k} \longrightarrow \mathbb{R} \\
\vec{x} \mapsto y_{0} \text { such that } f\left(\vec{x}, y_{0}\right)=0
\end{array}\right.
$$

Let $\mathcal{L}+! \mu$ be the set of functions defined by

$$
\mathcal{L}+! \mu=\left[0,1, U, \theta_{3} ; \mathrm{COMP}, \mathrm{CLI}, \mathrm{UMU}\right] .
$$

Lemma 3. $\mathcal{L} \subset \mathcal{L}+$ ! $\mu$.

Proof. (sketch) We only need to prove that constant functions -1 and $\pi$ are in $\mathcal{L}+$ ! $\mu$. Indeed, -1 is the unique root of $x \mapsto x+1$, and $\pi=4 \arctan (1)$, where $\arctan (x)$ is the solution of linear differential equation $\arctan (0)=0$ and $\arctan ^{\prime}(x)=\frac{1}{1+x^{2}}$, and $x \mapsto \frac{1}{1+x^{2}}$ can be obtained by applying UMU on $x, y \mapsto\left(1+x^{2}\right) y-1$.

Lemma 4. All functions from $\mathcal{L}+$ ! $\mu$ are of class $\mathcal{C}^{2}$ and total.

Proof. By structural induction. Basic functions $0,1, U, \theta_{3}$ are total and of class $\mathcal{C}^{2}$. Now, class $\mathcal{C}^{2}$ and totality are preserved by composition, by linear integration (see e.g. [1]), and by schema UMU by Lemma 2 .

Now, observe that operator UMU preserves real computability:

Lemma 5. Given $f: \mathbb{R}^{k+1} \longrightarrow \mathbb{R}$ real computable, if $\mathrm{UMU}(f)$ is defined, then $\mathrm{UMU}(f)$ is also real computable.

Proof. Given $\vec{x} \in \mathbb{R}^{k}$, let $y_{0}$ be the unique $y_{0}$ with $f\left(\vec{x}, y_{0}\right)=0$. Since $f(\vec{x},$. is continuous, non-decreasing, and with a unique root, we have $f(\vec{x}, y)<0$ for $y<y_{0}$, and $f(\vec{x}, y)>0$ for $y>y_{0}$.

There exists $m \in \mathbb{N}$, such that $f(\vec{x},-m)<0$ and $f(\vec{x}, m)>0$ : one just need to take any integer $m$ with $-m<y_{0}<m$. Actually, such an $m$ can be computed as follows: 
$m=1$

Repeat

Compute $f_{1}=f(\vec{x}, m)$ and $f_{2}=f(\vec{x},-m)$ at precision $\pm 2^{-m}$ $m=m+1$

Until $\left(f_{1}>2^{-m}\right.$ and $\left.f_{2}<-2^{-m}\right)$

Return $m$

Indeed, given any integer $m_{0} \in \mathbb{N}$ with $-m_{0}<y_{0}<m_{0}$, (take for example $\left.\left\lfloor\left|y_{0}\right|\right\rfloor+1\right)$, we have for all $m \geq m_{0}, f(\vec{x}, m) \geq f\left(\vec{x}, m_{0}\right)>0$ and $f(\vec{x},-m) \leq$ $f\left(\vec{x},-m_{0}\right)<0$. Now, for $m$ big enough (i.e. $m \geq m_{0}, 2^{-m} \leq\left|f\left(\vec{x},-m_{0}\right)\right|$, and $\left.2^{-m} \leq\left|f\left(\vec{x}, m_{0}\right)\right|\right)$ we have $f_{1}>2^{-m}$ and $f_{2}<-2^{-m}$ and the algorithm stops with an $m$ such that $f(\vec{x},-m)<0$ and $f(\vec{x}, m)>0$.

Computing $y_{0}$ then reduces to compute the unique root of function $f(\vec{x},$. over a compact $[-m, m]$. The fact that this is indeed computable can be seen as a consequence of the results in [31].

Here is a direct proof: given $n$, we have to find an approximation of $y_{0}$ at precision $2^{-n}$. Let us slice $[-m, m]$ in $2^{i}$ closed intervals: $[-m, m]=\cup_{0 \leq j<2^{i}}\left[y_{j}, y_{j+1}\right]$ where $y_{j}=-m+j \frac{2 m}{2^{i}}$. Let $z_{j}$ be an approximation of $f\left(\vec{x}, y_{j}\right)$ computed at precision $2^{-i}$. We know that for a root to exist in $\left[y_{j}, y_{j+1}\right]$, the only possibilities are that $\left|z_{j}\right|<2^{-i}$ or $\left|z_{j+1}\right|<2^{-i}$ or $z_{j} z_{j+1}<0^{3}$. Then, let $m_{i}$ be the $y_{j}$ (resp. $M_{i}$ be the $y_{j+1}$ ) where index $j$ is the smallest (resp. greatest) integer $0 \leq j<2^{i}$ with $\left|z_{j}\right|<2^{-i}$ or $\left|z_{j+1}\right|<2^{-i}$ or $z_{j} z_{j+1}<0$.

The sequences $\left(m_{i}\right)$ and $\left(M_{i}\right)$ have range in compact sets, so there exist subsequences $\left(m_{\phi(i)}\right)$ and $\left(M_{\phi(i)}\right)$ that converge, thanks to Bolzano-Weierstrass theorem. Let $m^{*}$ and $M^{*}$ be the limits of those sequences. For all $i$, either $\left|f\left(\vec{x}, m_{i}\right)\right| \leq\left|f\left(\vec{x}, m_{i}\right)-z_{j}\right|+\left|z_{j}\right|<2^{-i}+2^{-i}$, or $\left|f\left(\vec{x}, m_{i}+2^{-i}\right)\right| \leq \mid f\left(\vec{x}, m_{i}\right)-$ $z_{j+1}|+| z_{j+1} \mid<2^{-i}+2^{-i}$, or $f\left(\vec{x}, m_{i}\right) f\left(\vec{x}, m_{i}+2^{-i}\right)<0$. Since $f$ is continuous, we can deduce that $f\left(\vec{x}, m^{*}\right)=0$. For the same reason, $f\left(\vec{x}, M^{*}\right)=0$ and since $y \mapsto f(\vec{x}, y)$ has only one root, $m^{*}=M^{*}$. So, there exists $i$ such that $M_{i}-m_{i}<2^{-n}$. When this holds, $m_{i}$ is an approximation at precision $2^{-n}$ of the root. This means that the following algorithm terminates and returns an approximation of $y_{0}$ at precision $2^{-n}$.

$i=0$

Repeat

Compute $m_{i}$ and $M_{i}$

$i=i+1$

Until $M_{i}-m_{i}<2^{-n}$

Return $m_{i}$

Lemma 6. Given $h, g$ and c real computable, then $f=\operatorname{CLI}(g, h, c)$ is also real computable.

Proof. Observing carefully [10,9], if given $\vec{x} \in \mathbb{R}^{k}$ and some $\bar{y} \in \mathbb{Q}$ one can bound effectively the norms of $h(\vec{x}, y), f(\vec{x}, y), \frac{\partial^{2} f}{\partial y^{2}}(\vec{x}, y)$ for $|y| \leq \bar{y}$, then

\footnotetext{
${ }^{3}$ In fact, since the function we are investigating is non-decreasing, we could have more accurate constraints, however these ones are sufficient.
} 
$f$ will be real computable: use the constructions and bounds based on Euler's method to prove preservation of elementarily computability by linear integration in $[10,9]$, but replacing elementary bounds by computable bounds.

Now, from [31], it is known that one can bound effectively the norm of any real computable function on a compact domain, and so we only need to care about $f(\vec{x}, y)$ and $\frac{\partial^{2} f}{\partial y^{2}}(\vec{x}, y)$. But the norm of $f(\vec{x}, y)$ can be bounded effectively by Lemma 1 from bounds on the norms of $g(\vec{x})$ and $h(\vec{x}, y)$ on the corresponding domain, which are computable by previous argument. Now, $\left\|\frac{\partial^{2} f}{\partial y^{2}}(\vec{x}, y)\right\|=$ $\left\|\left(h^{2}(\vec{x}, y)+\frac{\partial h}{\partial y}(\vec{x}, y)\right) f(\vec{x}, y)\right\|$, hence is bounded by $\left.\left(\left\|h^{2}(\vec{x}, y)\right\|+\| c(\vec{x}, y)\right) \|\right) \times$ $\|f(\vec{x}, y)\|$. First factor can still be bounded effectively since $h^{2}(\vec{x}, y)$ and $c(\vec{x}, y)$ are particular real computable functions, and we just see that second factor can be bounded effectively.

From previous two Lemmas, the fact that basic functions are real computable and observing that composition is known to preserve real computability for total functions (see [31]), we obtain:

Theorem 1. Every function belonging to $\mathcal{L}+! \mu$ is real computable.

We now prove the converse direction. Following lemma is a weaker form of a Lemma that we proved in $[6]$ :

Lemma 7. Given $f: \mathbb{R}^{2} \rightarrow \mathbb{R}$ in $\mathcal{L}$, there exists $\tilde{f}: \mathbb{R}^{2} \rightarrow \mathbb{R}$ in $\mathcal{L}$ such that $\forall(m, n) \in \mathbb{N}^{2}, \forall(x, y) \in \mathbb{R}^{2}$,

- $\tilde{f}(m, n)=f(m, n)$

- $\tilde{f}(m, y) \in[f(m,\lfloor y\rfloor), f(m,\lfloor y+1\rfloor)]$ (or $[f(m,\lfloor y+1\rfloor), f(m,\lfloor y\rfloor)])$.

- $\tilde{f}(x, n) \in[f(\lfloor x\rfloor, n), f(\lfloor x+1\rfloor, n)]$ (or $[f(\lfloor x+1\rfloor, n), f(\lfloor x\rfloor, n)])$.

Proof. Let $\zeta=\frac{3 \pi}{2}$. Let $\omega: x \mapsto \zeta \theta_{3}(\sin (2 \pi x)) . \forall i, \int_{i}^{i+1} \omega=1$ and $\omega$ is equal to 0 on $\left[i+\frac{1}{2}, i+1\right]$ for $i \in \mathbb{N}$. Let $\Omega$ its primitive equal to 0 in 0 , and $i n t: x \mapsto \Omega\left(x-\frac{1}{2}\right)$. Function int is a function similar to the integer part: $\forall i \in \mathbb{N}, \forall x \in\left[i, i+\frac{1}{2}\right]$, $\operatorname{int}(x)=i=\lfloor x\rfloor$. Figure 1 shows graphical representations of $\omega$ and int.
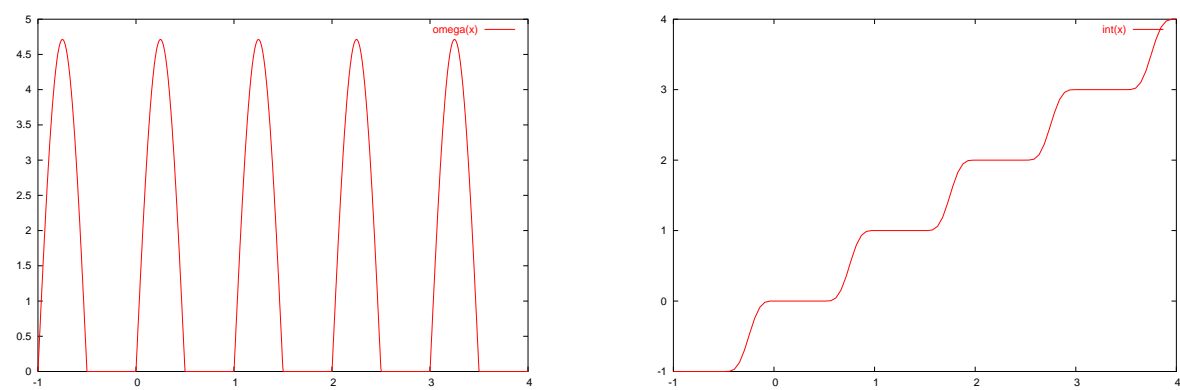

Figure 1. Graphical representation of $\omega$ and int 
Let $\Delta(i, y)=f(i, y+1)-f(i, y)$. Then for all $i \in \mathbb{N}, y \in \mathbb{R}$, we have $\omega(y) \Delta(i, \operatorname{int}(y))=\left\{\begin{array}{cl}0 & \text { whenever } y-\lfloor y\rfloor \geq 1 / 2 \\ \omega(y) \Delta(i,\lfloor y\rfloor) & \text { otherwise. }\end{array}\right.$

Let $G$ be the solution of the linear differential equation $G(x, 0)=f(x, 0)$, $\frac{\partial G}{\partial y}(x, y)=\omega(y) \Delta(x, \operatorname{int}(y))$. An easy induction on $j$ then shows that $G(i, j)=$ $f(i, j)$ for all integer $j$. Furthermore, by construction, $\forall i \in \mathbb{N}, G(i, y)$ belongs to the interval delimited by $G(i,\lfloor y\rfloor)=f(i,\lfloor y\rfloor)$ and $G(i,\lfloor y+1\rfloor)=f(i,\lfloor y+1\rfloor)$.

Now, let $\tilde{f}$ be the solution of the linear differential equation $\tilde{f}(0, j)=G(0, j)$, $\frac{\partial \tilde{f}}{\partial x}(x, y)=\omega(x)(G(\operatorname{int}(x+1), y)-G(\operatorname{int}(x), y))$. We have $\forall(i, j) \in \mathbb{N}^{2}, \tilde{f}(i, j)=$ $f(i, j)$. And $\forall i \in \mathbb{N}, \tilde{f}(i, y)$ belongs to the interval delimited by $\tilde{f}(i,\lfloor y\rfloor)=$ $f(i,\lfloor y\rfloor)$ and $\tilde{f}(i,\lfloor y+1\rfloor)=f(i,\lfloor y+1\rfloor)$. And also, $\forall j \in \mathbb{N}, \tilde{f}(x, j)$ belongs to the interval delimited by $\tilde{f}(\lfloor x\rfloor, j)=f(\lfloor x\rfloor, j)$ and $\tilde{f}(\lfloor x+1\rfloor, j)=f(\lfloor x+1\rfloor, j)$.

Theorem 2. Every recursive function over the integers has a real extension in $\mathcal{L}+! \mu$.

Proof. Let $\phi$ be a function from Rec. We have $\phi=\chi \circ ! \mu(\kappa)$ as in the proof of Proposition 2. Let $\iota(m, n)=2 \times(1 \ominus \sigma(m, n))+(1 \ominus \kappa(m, n))$ where $\sigma$ is the same as in the proof of Proposition 2. $\forall m \in \mathbb{N}$, for $n=n_{0}=! \mu(\kappa)(m, n)$, we have $\iota\left(m, n_{0}\right)=1$, and before this $n_{0}, \iota(m, n)$ is equal to 0 and after this $n_{0}, \iota(m, n)$ is equal to 2 . Let $i$ be a real extension of $\iota$ in $\mathcal{L}$ given by Proposition 5 . Let $\tilde{i}$ be the function from $\mathcal{L}$ obtained by Lemma 7 on $f(m, x): m, x \mapsto i(m, x)-1$.

$\forall m \in \mathbb{N}$, there exists exactly one $y \in \mathbb{R}$ (given by $y_{0}=! \mu(\kappa)(m, n)$ ) such that $\tilde{i}(m, y)=0$. But, we can not directly apply schema UMU, since we have no assurance $^{4}$ that it also holds for non integer values $m$. However, from the constructions in the proof of Lemma 7 , given $m \in \mathbb{N}$, we have $\tilde{i}(m, y)$ equal to -1 for $y \leq y_{0}-1$, and equal to $\Omega(y)$ for $y \in\left[y_{0}-1, y_{0}+1\right]$, where $\Omega$ is defined in that proof.

Consider $\mathcal{M}(x)=\theta_{3}(x+1)$. We have $\mathcal{M}(x)=0$ if $x \leq-1$ and $\mathcal{M}(x) \geq 1$ if $x \geq 0$. Let us define $\tilde{g}$ as the solution of the differential equation $\tilde{g}(\vec{x}, 0)=-1$, $\frac{\partial \tilde{g}}{\partial y}(\vec{x}, y)=\alpha \mathcal{M}(\tilde{i}(\vec{x}, y))$. Let us choose $\alpha$ (maple says $\left.\alpha=\frac{1024}{2609}\right)$ such that $\alpha \int_{-1}^{0} \mathcal{M}(\Omega(x)) \mathrm{d} x=1$. We have $\forall m \in \mathbb{N}, \tilde{g}(m, y)=0 \Leftrightarrow y=! \mu(\kappa)(m, n)$.

Then define $g$ as the solution of the linear differential equation $g(\vec{x}, 0)=-1$, $\frac{\partial g}{\partial y}(\vec{x}, y)=\beta \mathcal{M}(\tilde{g}(\vec{x}, y))$. If we choose $\beta$ adequately ${ }^{5}$ (maple says $\beta=\frac{a \pi^{4}}{b \pi^{4}-c \pi^{2}+d}$ for some integers $a, b, c, d)$, we will still have $\forall m \in \mathbb{N}, g(m, y)=0 \Leftrightarrow y=$ $! \mu(\kappa)(m, n)$.

The point is that, since $\mathcal{M}$ is always non-negative, we know that $\forall x \in \mathbb{R}$, $y \mapsto \tilde{g}(x, y)$ is non-decreasing, and, because of Lemma 7, and from the definition of function $\mathcal{M}(x)$, it must go to infinity when $y$ goes to infinity. Actually, it must be equal to -1 up to a certain value $y_{-}$, then be strictly increasing, and since it goes to infinity, it must have a root $y_{0}$ strictly greater than $y_{-}$. Now the derivative in this root $y_{0}$ cannot be 0 since $\mathcal{M}(x)$ is zero only when $x \leq-1$.

\footnotetext{
4 Actually, another problem is that the derivative relative to the second variable in the root point is 0 .

${ }^{5}$ This $\beta$ is in $\mathcal{L}$ since it can be obtained as $a * \pi^{4} * \operatorname{UMU}\left(x \mapsto\left(b \pi^{4}-c \pi^{2}+d\right) x-1\right)$.
} 
This $g$ is such that $\forall \vec{x}, \exists ! y_{0}$ such that $g\left(\vec{x}, y_{0}\right)=0$ and $\frac{\partial g}{\partial y}\left(\vec{x}, y_{0}\right) \neq 0$ and for all $\vec{x}, y \mapsto g(\vec{x}, y)$ is non-decreasing. We can thus apply UMU to this $g$. Now if we extend $\chi$ in a real function $h$ belonging to $\mathcal{L}$ using Proposition 5, we have $h \circ \mathrm{UMU}(g)$ extending $\phi=\chi \circ \mu(\psi)$ and belonging to $\mathcal{L}+! \mu$.

From previous two theorems, we obtain the main result of this paper:

Theorem 3. $\mathcal{R} e c=D P(\mathcal{L}+! \mu)$. I.e:

- If a function from $\mathcal{L}+! \mu$ extends some function over the integers, this latter function is total recursive.

- Any total recursive function over the integers, has a real extension that belongs to $\mathcal{L}+! \mu$.

Proof. The second item is Theorem 2. The first item is immediate from Theorem 1 : if a function $f$ belonging to $\mathcal{L}+! \mu$ preserves integers, then a recursive function that equals $f$ on $\mathbb{N}^{k}$ can easily be obtained from the functional computing $f$.

Corollary 1. $\mathcal{L}$ is strictly included in $\mathcal{L}+! \mu$.

\section{References}

1. V. I. Arnold. Ordinary Differential Equations. MIT Press, 1978.

2. E. Asarin and A. Bouajjani. Perturbed Turing machines and hybrid systems. In Logic in Computer Science, pages 269-278, 2001.

3. E. Asarin and O. Maler. Achilles and the tortoise climbing up the arithmetical hierarchy. Journal of Computer and System Sciences, 57(3):389-398, dec 1998.

4. O. Bournez. Achilles and the Tortoise climbing up the hyper-arithmetical hierarchy. Theoretical Computer Science, 210(1):21-71, 61999.

5. O. Bournez. Complexité Algorithmique des Systèmes Dynamiques Continus et Hybrides. PhD thesis, Ecole Normale Supérieure de Lyon, Janvier 1999.

6. O. Bournez and E. Hainry. An analog characterization of elementary computable functions over the real numbers. In International Colloquium on Automata, Languages and Programming (ICALP 2004), volume 3142 of Lecture Notes in Computer Science, pages 269-280, 2004.

7. V. Brattka. Recursive characterizations of computable real-value functions and relations. Theoretical Computer Science, 162(1):45-77, 5 August 1996.

8. V. Brattka. Computability over topological structures. In S. B. Cooper and S. S. Goncharov, editors, Computability and Models, pages 93-136. Kluwer Academic Publishers, New York, 2003.

9. M. Campagnolo, C. Moore, and J. F. Costa. An analog characterization of the Grzegorczyk hierarchy. Journal of Complexity, 18(4):977-1000, 2002.

10. M. L. Campagnolo. Computational complexity of real valued recursive functions and analog circuits. PhD thesis, Universidade Técnica de Lisboa, 2001.

11. G. Etesi and I. Németi. Non-Turing computations via Malament-Hogarth spacetimes. International Journal Theoretical Physics, 41:341-370, 2002.

12. D. Graça and J. F. Costa. Analog computers and recursive functions over the reals. Journal of Complexity, 19:644-664, 2003. 
13. A. Grzegorczyk. Computable functionals. Fundamenta Mathematicae, 42:168-202, 1955.

14. T. Henzinger and J.-F. Raskin. Robust undecidability of timed and hybrid systems. Hybrid Systems: Computation and Control; Second International Workshop, HSCC'99, Berg en Dal, The Netherlands, march 29-31, 1999; proceedings, 1569, 1999.

15. M. L. Hogarth. Does general relativity allow an observer to view an eternity in a finite time? Foundations of Physics Letters, 5:173-181, 1992.

16. L. Kalmár. Egyszerü példa eldönthetetlen aritmetikai problémára. Mate és fizikai lapok, 50:1-23, 1943.

17. D. Lacombe. Extension de la notion de fonction récursive aux fonctions d'une ou plusieurs variables réelles III. Comptes rendus de l'Académie des Sciences Paris, 241:151-153, 1955.

18. L. Lipshitz and L. A. Rubel. A differentially algebraic replacement theorem, and analog computability. Proceedings of the American Mathematical Society, 99(2):367-372, February 1987.

19. C. Moore. Recursion theory on the reals and continuous-time computation. Theoretical Computer Science, 162(1):23-44, 51996.

20. J. Mycka. Infinite limits and R-recursive functions. Acta Cybernetica, 16:83-91, 2003.

21. J. Mycka. $\mu$-recursion and infinite limits. Theoretical Computer Science, 302:123$133,2003$.

22. P. Odifreddi. Classical recursion theory II. North-Holland, 1999.

23. T. Ord. Hypercomputation: computing more than the Turing machine. Technical report, University of Melbourne, september 2002. See http://www.arxiv.org /abs/math.lo/0209332.

24. P. Orponen. A survey of continuous-time computation theory. In D.-Z. Du and K.I. Ko, editors, Advances in Algorithms, Languages, and Complexity, pages 209-224. Kluwer Academic Publishers, Dordrecht, 1997.

25. M. B. Pour-El. Abstract computability and its relation to the general purpose analog computer (some connections between logic, differential equations and analog computers). Transactions of the American Mathematical Society, 199:1-28, 1974.

26. E. Ramis, C. Deschamp, and J. Odoux. Cours de mathématiques spéciales, tome 3, topologie et éléments d'analyse. Masson, feb 1995.

27. H. Rose. Subrecursion: functions and hierarchies. Clarendon Press, 1984.

28. C. E. Shannon. Mathematical theory of the differential analyser. Journal of Mathematics and Physics MIT, 20:337-354, 1941.

29. H. Siegelmann. Neural networks and analog computation - beyond the Turing limit. Birkauser, 1998.

30. A. Turing. On computable numbers, with an application to the "Entscheidungsproblem". In Proceedings of the London Mathematical Society, volume 2, pages 230-265, 1936.

31. K. Weihrauch. Computable analysis. Springer, 2000.

32. Q. Zhou. Subclasses of computable real valued functions. Lecture Notes in Computer Science, 1276:156-165, 1997. 